\title{
ESTIMATION OF PROTHROMBIN TIME IN PREGNANCY COMPARED WITH NORMAL CONTROLS
}

Pannala Srimala, Inayatulla Khan, Puli Sree Hari

1. Tutor, Department of Physiology, Rajiv Gandhi Institute of Medical Sciences, Adilabad, Andhra Pradesh.

2. Tutor, Department of Physiology, Rajiv Gandhi Institute of Medical Sciences, Adilabad, Andhra Pradesh.

3. Associate Professor, Department of Physiology, Rajiv Gandhi Institute of Medical Sciences, Adilabad, Andhra Pradesh.

\section{CORRESPONDING AUTHOR}

Dr. Mohd Inayatulla Khan, Department of Physiology, Rajiv Gandhi Institute of Medical Sciences, Adilabad, Andhra Pradesh, E-mail: drkhan123@rediffmail.com Ph: 00919948959937.

ABSTRACT: BACKGROUND: Normal pregnancy is associated with substantial changes in the tissue factor pathway and in the wider haemostatic system [1]. It is also characterized by impressive changes in the activating and inhibitory pathways of coagulation and fibrinolysis resulting in an accelerated, but well balanced, process of thrombin formation and resolution. These changes serve to protect the mother from the hazard of bleeding imposed by placentation and delivery, but they also carry the risk of an exaggerated response, localized or generalized, to coagulant stimuli [2]. Hemorrhage occupies an important position in the etiology of maternal mortality and therefore, remains a major problem [3]. To what extent normal pregnancy affects coagulation is not well known in our locality. Thus, our study aims to find out the changes that occur in the coagulation parameters in pregnancy as compared to that in normal controls.

METHODS: The study population included 25 healthy pregnant women, who visited the antenatal clinic of the KIMS Hospital in Narketpally, Nalgonda AP, between October 2008 and May 2009. 25 healthy age-matched non-pregnant women served as controls for the study. Both subjects and controls were randomly chosen from general population of pregnant women. Prothrombin Time test was done by using reagents bought from "UNIPLASTIN", TULIP DIAGNOSTICS (P) LTD. When UNIPLASTIN reagent is added to normal citrated plasma, the clotting mechanism is initiated, forming a solid gel clot within a specified period of time. RESULTS: The results showed that the control group has a mean Prothrombin Time of $9.16 \mathrm{sec}$ with SD of \pm 1.10 where as the test group showed a mean Prothrombin Time of 14.32 Sec and SD of $\pm 1.91, P<0.001$ by the chi-square test is significant. Whereas the International Normalized Ratio INR for control group was $1.26 \mathrm{Sec}$ and SD of $\pm 0.15 \mathrm{Sec}$ and the test group had INR of 1.67 Sec and SD of $\pm 0.26 \mathrm{Sec}$, the $\mathrm{p}$ value is $<0.96$ by chi-square test is insignificant. The odds ratio for Prothrombin Time was 1.73 which indicates that the Prothrombin Time was significantly decreased in the test group. CONCLUSION: The fact that Prothrombin time is decreased in normal pregnancy should be interpreted with caution, and that INR is the better indicator of the coagulation profile of the individual, therefore Prothrombin Time should always be read with INR ratio.

KEY WORDS: PT - Prothrombin Time, INR- International Normalized Ratio 
INTRODUCTION: Haemostasis in normal pregnancy involves a complex network of interactions with positive and negative feedback loops, integrating blood vessels; platelets, coagulation factors, coagulation inhibitors and fibrinolysis and has evolved to maintain the integrity of the vasculature. Normal pregnancy is associated with substantial changes in the tissue factor pathway and in the wider haemostatic system [1]. Normal pregnancy is a characterized by impressive changes in the activating and inhibitory pathways of coagulation and fibrinolysis resulting in an accelerated, but well balanced, process of thrombin formation and resolution. These changes serve to protect the mother from the hazard of bleeding imposed by placentation and delivery, but they also carry the risk of an exaggerated response, localized or generalized, to coagulant stimuli [2]. Hemorrhage occupies an important position in the etiology of maternal mortality and therefore, remains a major problem [3]. There is activation of blood coagulation and a simultaneous increase in fibrinolysis without signs of organ dysfunction during normal pregnancy. These changes increase as pregnancy progresses. During delivery there is consumption of platelets and blood coagulation factors including fibrinogen [4].

Pregnancy is a risk factor for venous thrombosis and the incidence of venous thromboembolism during normal pregnancy is 6-fold higher than in the general female population of child bearing age. Venous thromboembolism is an important cause of maternal morbidity and mortality [5].

The coagulation cascade is in an activated state in pregnancy. Activation includes increased concentrations of all clotting factors, except factors XI, XIII, with increased levels of High molecular weight fibrinogen complexes.

Changes in the haemostatic mechanism also involve decreased levels of anticoagulant proteins such as protein $\mathrm{C}$ and Protein $\mathrm{S}$ as well as enhanced thrombin generation and decreased fibrinolytic activity ${ }^{[6]}$.

AIMS: To what extent normal pregnancy affects coagulation is not well known. A study like this is therefore necessary to assess the influence of normal uncomplicated pregnancy on Prothrombin time and also to know, the extent of relationship between pregnancy and Prothrombin time.

1. To study changes in Prothrombin time in normal pregnancy.

2. To study the Prothrombin time in normal age matched controls

3. To assess the changes in Prothrombin time between normal and pregnant females.

MATERIALS AND METHODS: SUBJECTS: The study population included 25 healthy pregnant women in third trimester, who visited the antenatal clinic of the KIMS Hospital in Narketpally, Nalgonda from October 2008 to May 2009.

25 healthy age-matched non-pregnant women served as controls for the study. Both subjects and controls were randomly chosen from general population of pregnant women.

The study was approved by college ethics committee and all subjects gave written consent to participate in the study voluntarily.

INCLUSION CRITERIA:

Study is subdivided into two groups 


\section{Controls}

a) Healthy females age above 20 and below 35 years

b) No history of significant medical illness.

c) Individuals who agreed to participate for the study voluntarily.

2. Test group (pregnant).

a) Healthy females age above 20 and below 35 years

b) 25 healthy pregnant women in third trimester were taken as study group.

c) No history of significant gynecological problems.

\section{EXCLUDING CRITERIA:}

i. $\quad$ Subjects $<20$ years and $>35$ years

ii. Cardiovascular diseases

iii. Renal diseases

iv. Liver diseases,

v. Endocrinal disorders

MATERIALS: Prothrombin time test was done by using reagents bought from "UNIPLASTIN", TULIP DIAGNOSTICS (P) LTD.

UNIPLASTIN is a novel, highly sensitive, low opacity, ready to use liquid Calcified Thromboplastin Reagent, which is derived from rabbit brain.

PRINCIPLE: Tissue thromboplastin in the presence of calcium activates the extrinsic pathway of human blood coagulation mechanism. When UNIPLASTIN reagent is added to normal citrated plasma, the clotting mechanism is initiated, forming a solid gel clot within a specified period of time.

SAMPLE COLLECTION: Patients were advised against vigorous exercises before sample collection, fasting (or after light fatty meals) was collected.

\section{PROCEDURE:}

- $1.8 \mathrm{ml}$ of venous blood was collected under sterile conditions and immediately added to $0.2 \mathrm{ml}$ of $3.2 \%$ tri sodium citrate (anticoagulant).

- Centrifuge immediately for $15 \mathrm{~min}$ at $1500-2000 \mathrm{rpm}$ and transfer the plasma into a clean test tube.

- It should be ensured that the plasma is free from platelets (PPP). Cap the test tubes to prevent deterioration of samples. Plasma must be tested preferably immediately.

- Bring the reagent vial to room temperature $\left(20-30^{\circ} \mathrm{C}\right)$. Mix the contents of the vial to homogenize the suspension completely.

- Aspirate from the reagent vial enough reagents for immediate testing requirements in a thoroughly clean and dry test tube.

- Pre-warm the reagent and bring to $37^{\circ} \mathrm{C}$ before use in test procedure

- Recap the reagent vial and replace immediately to $2-8^{\circ} \mathrm{C}$.

- To a $12 \times 75 \mathrm{~mm}$ tube add $0.1 \mathrm{ml}$ of plasma and place the tube in a water bath for 35 minutes at $37^{\circ} \mathrm{C}$.

- To the tube forcibly add $0.2 \mathrm{ml}$ of UNIPLASTIN reagent and simultaneously start a stopwatch. Shake the tube gently to mix contents. 
- Gently tilt the tube back and forth and stop the stopwatch as soon as the first fibrin strand is visible and the gel/clot formation begins. Record the time in 'seconds'.

- Repeat steps above for a duplicate test on the same sample.

- Find the average of the duplicate test values. This is the Prothrombin Time (PT).

\section{CALCULATION OF RESULTS}

\section{Manual Method}

The results may be reported directly in terms of the mean of the double determination of PT of the test plasma in 'seconds'. Or as a ratio 'R':

\section{$R=\quad$ Mean of the patient plasma PT in seconds MNPT for the reagent}

Or as International Normalized Ratio (INR), INR = (R) ISI, where ISI = International Sensitivity Index of the reagent

It is recommended by the WHO that MNPT should be established for each lot of PT reagents by each laboratory, since PT results are dependent on the combination of reagent lot, instrument and technique followed at each laboratory. Usually plasma from at least 20 normal healthy individuals should be used to establish the MNPT. The average of such PT results in seconds $=$ MNPT .

\section{EXPECTED VALUES}

Normal values using UNIPLASTIN $®$ are between 11-15 seconds.

RESULT PT: The results showed that the control group has a mean Pro thrombin Time of 9.16 sec with SD of \pm 1.10 where as the test group showed a mean Pro thrombin Time of 14.32 Sec and SD of $\pm 1.91, P<0.001$ by the chi-square test which is significant. Whereas the International Normalized Ratio INR for control group was $1.26 \mathrm{Sec}$ and SD of $\pm 0.15 \mathrm{Sec}$ and the test group had INR of $1.67 \mathrm{Sec}$ and SD of $\pm 0.26 \mathrm{Sec}$, the $\mathrm{p}$ value is $<0.96$ by chi-square test is insignificant. The odds ratio for Pro Thrombin Time was 1.73 which indicates that the Pro Thrombin Time was significantly increased in the test group.

DISCUSSION: In the present study there is decreased Prothrombin time during normal pregnancy when compared with age matched control groups of non pregnancies. This decreased Prothrombin time may be because of change in haemostatic balance in the direction of Hypercoagulability in which increase concentration of all clotting factors except XI, XIII.

One similar done by Nihon Sanka et al; showed that in the third trimester, shortening of prothrombin time and activated partial thromboplastin time [11]. Hellgren M reported Increased endogenous thrombin generation, acquired activated protein $\mathrm{C}$ resistance and increased prothrombin complex level (PT) measured as international normalized ratio (INR) of less than 0.9 have been reported as well [4].

One similar study in which they also measured Prothrombin fragments, by Cerneca F et al has shown that the parameters showing the greatest variation during pregnancy were PT, FBG, PS, Prothrombin fragments F1+2. The existence of a hypercoagulable state in pregnancy was suggested by the increased levels of F1+2 [12]. 
According to a previous study prolonged Prothrombin time shows fetal weight gain from the $2^{\text {nd }} \& 3^{\text {rd }}$ trimesters [1].

Lloyd R et al; showed that Prothrombin time was also decreased in pregnancy and Pregnancy was associated with a significant increase in the activity of factors VII, VIII, IX, and X and in the concentrations of fibrinogen, $\mathbf{x}$-1-globulin, and $\alpha$-1-antitrypsin [8]. Yet another study showed that term pregnant women were in a hypercoagulable state and had increased fibrinolysis [13].

In one study by Hui C, Lili $\mathrm{M}$ et al; the TEG Prothrombin time, activated partial thromboplastin time, thrombin time, international normalized ratio, and thrombomodulin and resistance index in uterine arteries showed a tendency to decrease in pregnant women [14].

Some comparative similar studies also shown, Prothrombin fragments are increased and Prothrombin time is decreased in pregnancy [7-10].

One study by Jørgensen M, Klajnbard A et al; has shown that Prothrombin time remains unchanged in pregnancy, which was contrary to our findings they also showed that the level of coagulation factors II, V, X, XI, XII and antithrombin, protein C largely remained unchanged [15].

The INR was not affected significantly in this study. The INR was introduced in the early 1980s when it turned out that there was a large degree of variation between the various Prothrombin time assays, a discrepancy mainly due to problems with the purity of the thromboplastin (tissue factor) concentrate. The INR became widely accepted worldwide, especially after endorsement by the World Health Organization. Since INR did not show any significant increase in the test group there is no any significant bleeding possibilities in the normal pregnancy.

SUMMARY AND CONCLUSION: Within the limitation of our study it is concluded that Prothrombin time is decreased in normal pregnancy but it should be interpreted with caution, and that INR is the better indicator of the coagulation profile of the individual, therefore Prothrombin Time should always be read with INR ratio.

The INR is a method of expressing the results of a Prothrombin time blood test. It is based on an international standard that automatically corrects for variations between labs.

Thus, using the INR, the PT measurement from one lab can be compared to a PT measurement from any other lab in the world, even if they use different methods to measure PT. the resulting measurement is often referred to as the INR/PT.

\section{BIBLIOGRAPHY:}

1. F.I.Buseri, Z.A.Jeremaiah and F.G.Kalio - Influence of Pregnancy and Gestation Period on Some Coagulation Parameters among Nigerian Antenatal Women. Research Journal of Medical Sciences 2(6):275-281, 2008.

2. Bijoy Sree Sengupta, Sisir K.Chattopadyay, Hanes G.Thornton, Partha Sarathi Sengupte - Obstetrics for Post graduates \& Practitioners Aspects of Fetal and Maternal Medicine 1st Edition 1999, B.I.Churchill Livingstone Pvt. LTD, pp 4-10.

3. Agarwal S, Asha Buradkar.Coagulation studies in toxaemias of pregnancy. Journal of Obstetrics and Gynaecology of India 1978; 992-996.

4. Hellgren M. Hemostasis during normal pregnancy and puerperium. Semin Thromb Hemost.2003 Apr; 29(2); 125-30. 
5. James AH, Bushnell CD, Jamison MG, Myers ER. Incidence and risk factors for stroke in pregnancy and the puerperium. Obstet Gynecol. 2005; 106: 509-516.

6. Dati F, Pelzer H, Wagner C. Changes in the haemostatic Relevance of markers of Hemostasis activation in obstetrics/gynecology and pediatrics. Dade Behring, Marburg, Germany. PMID:9834011

7. Federico Cerneca, Giuseppe Ricci, Roberto Simeone, et al, European Journal of Obstetrics \& Gynecology and Reproductive Biology, Volume 73, Issue 1, Pages 1-110 (May 1997); 31-36.

8. Lloyd R Whitfield, Amol S Lele, Gerhard Levy Amherst et al, Effect of Pregnancy on the relationship between concentration and anticoagulant action of heparin. Clinical Pharmacology and Therapeutics (1983) 34, 23-28;1983.

9. Uchikova EH, Ledjev II - Changes in haemostasis during normal pregnancy. Eur J Obstet Gynecol Reprod Biol.2005 Apr 1; 119(2):185-8.

10. Usta IM - Acute fatty liver of pregnancy: an experience in the diagnosis and management of fourteen cases. Am J Obstet Gynecol - 01-NOV-1994; 171(5):13427.

11. Nihon Sanka Fujinka Gakkai Zasshi. A study of intrapartum and postpartum bleeding based on changes in blood coagulation and fibrinolysis during pregnancy. [Article in Japanese] 1987 Mar;39(3):405-10. PMID: 3494090

12. Cerneca F, Ricci G, Simeone R, et al 'Coagulation and fibrinolysis changes in normal pregnancy. Increased levels of procoagulants and reduced levels of inhibitors during pregnancy induce a hypercoagulable state, combined with a reactive fibrinolysis' Eur J Obstet Gynecol Reprod Biol. 1997 May;73(1):31-6.

PMID:9175686

13. Go GH, Lee SK, Son JS. Assessment of Coagulation Profiles in Healthy Term Pregnant Women Using a Thrombelastography. Korean I Anesthesiol. 2002 Jun;42(6):776782.

14. Hui $\mathrm{C}$, Lili $\mathrm{M}$, et al; Changes in coagulation and hemodynamics during pregnancy: a prospective longitudinal study of 58 cases. Arch Gynecol Obstet. 2012 May;285(5):1231-6. doi: 10.1007/s00404-011-2137-x. Epub 2011 Nov 15. PMID: 22083312

15. Szecsi PB Jørgensen M, et al; Haemostatic reference intervals in pregnancy, Thromb Haemost. 2010 Apr; 103(4):718-27. doi: 10.1160/TH09-10-0704. Epub 2010 Feb 19. PMID: 2017476. 
RESULT PT:

\begin{tabular}{|l|l|l|l|l|l|}
\hline & $\begin{array}{c}\text { CONTROL } \\
\text { GROUP }\end{array}$ & TEST GROUP & t-VALUE & P-VALUE & RESULT \\
\hline $\begin{array}{l}\text { PROTHROMBIN } \\
\text { TIME }\end{array}$ & $9.16 \pm 1.10$ & $14.32 \pm 1.91$ & 2.05 & $<0.001$ & SIGNIFICANT \\
\hline $\begin{array}{l}\text { INTERNATIONAL } \\
\text { NORMALIZED } \\
\text { RATIO(INR) }\end{array}$ & $1.26 \pm 0.150$ & $1.67 \pm 0.26$ & 9.55 & $<0.965$ & INSIGNFICANT \\
\hline
\end{tabular}
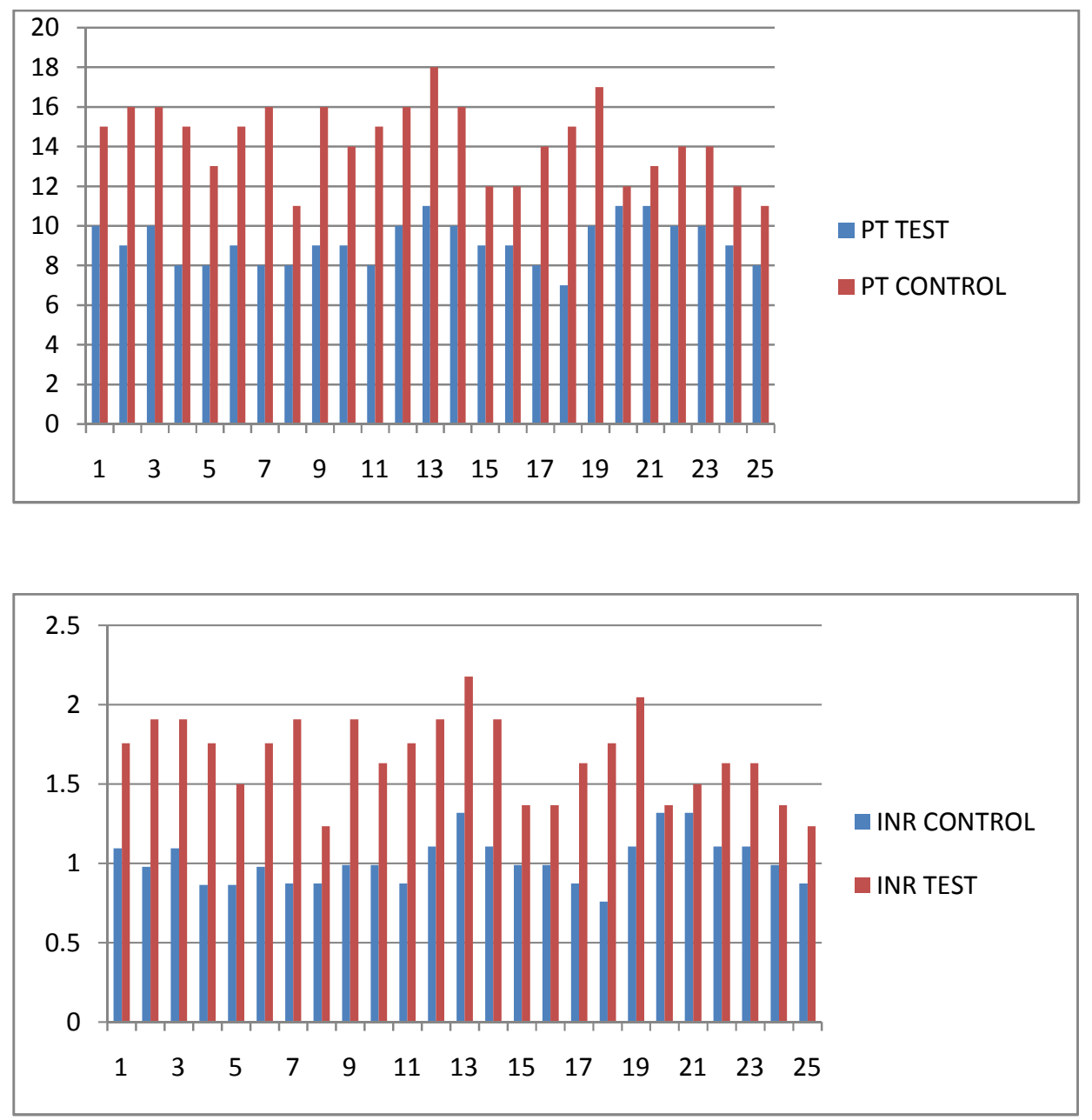